\title{
Assistive Technology Programs to Support Persons with Neurodevelopmental Disorders
}

\author{
Giulio E. Lancioni ${ }^{1}$ \\ Published online: 28 July 2018 \\ (C) Springer Nature Switzerland AG 2018
}

\begin{abstract}
Assistive technology is increasingly popular within different education, rehabilitation, and care contexts, and it includes a variety of technology solutions (Puanhvuan et al. 2017; Schlosser et al. 2017; Williamson et al. 2017; Wu et al. 2016). The variety is basically due to the different groups of individuals with whom it is used and the different objectives pursued with them. Indeed, literature has shown that a wide range of individuals with different disability histories, needs, and education/ rehabilitation or care goals (e.g., individuals with multiple disabilities due to prenatal or perinatal causes, individuals with neurodegenerative disease, and individuals with acquired brain damage) can benefit from the use of technology-aided programs (Boyd et al. 2017; Lancioni and Singh 2014).
\end{abstract}

\section{Examples of Assistive Technology Solutions and Related Programs}

Individuals with Multiple Disabilities Individuals with severe intellectual disability, lack of communication, and extensive motor impairment may be unable to have any contact with their immediate environment. Any chance for them to establish such contact and develop basic forms of control of preferred environmental stimulation would depend on the possibility of using the minimal response repertoire they possess. The technology-aided program required to help these individuals might involve (a) a microswitch (sensor) that the individuals can activate with a small response (e.g., a small head, finger, lip, or eyelid movement) and (b) a computer or other electronic device that responds to microswitch activations by delivering specific stimulation. The use of this technologyaided program can provide the individuals with the necessary

Giulio E. Lancioni

giulio.lancioni@uniba.it

1 Department of Neuroscience and Sense Organs, University of Bari, Corso Italia 23, 70121 Bari, Italy support to make their small responses functional to access and control preferred environmental stimulation (Lancioni and Singh 2014; Mechling 2006).

Individuals with intellectual and visual disabilities may tend to be sedentary and fail to engage in activities requiring mobility. Providing direct staff guidance might be practically difficult and may also be detrimental for the individuals' social image because it emphasizes their dependence. A technologyaided program suitable to help them overcome their problems could rely on the use of (a) sound boxes placed in different areas of their activity context to provide space guidance (orientation) to those areas, and (b) a computer or other system that can regulate (i.e., activate and deactivate) those boxes and provide positive stimulation in relation to the participants' arrival at the destinations and/or following their activities (Lancioni et al. 2018c).

Individuals with Neurodegenerative Disease The neurodegenerative disease with the greatest social impact is the Alzheimer's disease. Individuals affected by this disease face a process of deterioration of their functions with no possibility of preventing or halting it. A viable way of helping these individuals is to provide them with assistive technology solutions aimed at alleviating the impact of the disease. For example, individuals at the mild stage of the disease may have problems remembering the different activities to carry out during the day as well as the steps involved in the activities. A technologyaided program to help counter these problems may involve the use of a commercial device, such as a tablet or smartphone, which is programmed to alert the individuals about the activities (i.e., at the appropriate times) and to provide them verbal instructions/cues concerning the steps to carry out. Actually, the individuals may receive all the required inputs via a wireless Bluetooth earpiece linked to the tablet or smartphone (Kerkhof et al. 2016; Lancioni et al. 2017; Wang et al. 2017).

Individuals at the severe or low-moderate stages of the disease present extensive disabilities that curb their occupational opportunities and frequently also their ambulation. 
Indeed, many of these persons are no longer able to walk unsupported and spend their time in wheelchairs. Any attempt to help them cannot only rely on the use of walker devices. In fact, the problem might be due to motor weakness as well as to lack of motivation. One way to deal with the problem is to combine the use of a walker with (a) microswitches to monitor the individuals' steps and (b) an electronic device programmed to record those steps and follow them with brief periods of preferred stimulation. For example, the individuals could receive $3-5 \mathrm{~s}$ of preferred music at each step so regular ambulation would ensure that they receive uninterrupted music (Lancioni et al. 2018d).

Individuals with Acquired Brain Damage Individuals who have a diagnosis of minimally conscious state combined with pervasive motor impairment pose a very severe challenge to any professional in the field. These individuals could benefit from a technology-aided program that allows their responding (albeit limited) to link them to preferred stimulation or to call the attention of staff or caregivers. The technology required might involve a speech-generating device (SGD) and a microswitch connected to a computer system (Lancioni et al. 2014). The microswitch would allow them independent access to preferred environmental stimulation. The SGD would allow them to verbally request (irrespective of their lack of verbal skills) the attention of the caregiver or staff members. In fact, the SGD would emit a verbal request for such attention in relation to a simple motor response of the individual.

Individuals who have emerged from a minimally conscious state but present motor and communication impairments might use a more advanced technology-aided program. Such program could rely on a computer system presenting leisure and communication options (e.g., music, telephone calls, messages, and videos) and a microswitch. The microswitch would allow the individuals to select any of the options presented by the computer system. Once an option is selected, the individual is allowed to make choices within that option (e.g., can choose among different songs and among different partners to reach via telephone call or text message) (Lancioni et al. 2014).

\section{Articles Included in This Special Issue}

Ten articles are included in this special issue. They represent new efforts to examine the suitability and possible impact of assistive technology solutions and technology-aided intervention programs for individuals with neurodevelopmental disorders (i.e., mental illness, intellectual disability, autism spectrum disorder, intellectual and motor or sensory-motor disabilities, traumatic brain injury, and minimally conscious state combined with extensive motor impairment). Specifically, Beale et al. (2018) conducted a two-phase evaluation of a multi-user private virtual world "Aftercare World" accessible from mid-range computers or tablets. Phase I involved the development of an initial prototype virtual world and the survey of 46 support workers to determine their views about that world. Phase 2 involved the development of a second prototype and its evaluation by 20 support workers and 10 clients from a mental health support service following an instruction session with the prototype. Results were mixed for the initial prototype but positive for the second one. Indeed, most of the support workers and clients found it easy to use the virtual world features of the second prototype when assisted by a trainer, and most support workers indicated that they would use it for their own learning and to assist some of their clients.

Myers et al. (2018) assessed the effectiveness of telehealth technology to train parents of 32 adolescents or young adults on a mindfulness-based health wellness (MBHW) program that they were then to teach to their children. The 32 children (i.e., adolescents and young adults) were overweight or obese and presented with a mild level of intellectual disability. The effects of the program on the children's body weight were assessed within a changing criterion design. The intervention included physical exercise, healthy eating and nutrition, mindful eating, mindful response to thoughts of hunger, and a mindfulness practice to control the urge to eat. The 30 children who went through the program successfully had an average weight reduction of $38.27 \mathrm{lb}$. by the end of intervention and maintained their new weight for 4 consecutive years. Within a social validity rating, the children also reported great satisfaction with the program effects.

Davies et al. (2018) assessed a technology solution (i.e., GeoTalk) consisting of a handheld device application that integrates global positioning system (GPS) and other sensor technologies to automatically switch between vocabulary sets when the user enters a designated geographic zone, such as a school, bank, and grocery store. Once a GPS communication zone was entered, the user was provided with the corresponding vocabulary set so that requests pertaining to that zone could be made. A change would occur automatically when the user moved to a new zone. The new zone would be announced and the user could automatically access a new vocabulary set pertaining to the new zone. The study involved 37 participants with mild to moderate intellectual disability. The participants were able to operate the GPS-enabled GeoTalk device with fewer errors and prompts, and with greater speed than two commercially available comparison AAC devices.

Damianidou et al. (2018) extended a recently published meta-analysis on the impact of technology to support employment-related outcomes of people with intellectual and developmental disabilities by analyzing the types of technology and work settings used. Regarding the types of technology, visible effect differences were found between pictorial prompts (least effective) and alternatives such as auditory prompting devices, desktop and laptop computers, and palmtops. Regarding the setting, findings showed that the 
interventions taking place in real work settings had a significantly lower average outcome than those taking place in simulated work settings. Significant relationships were also found between the presence of universal design features and types of technology. Palmtops, and desktop and laptop computers, for example, included the use of universal design features more frequently than pictorial prompts. The implications of those findings for new research in the area were discussed.

Desideri et al. (2018) carried out a preliminary evaluation of a social robot within a public child and adolescent mental health service. Their goal was to determine whether the robot could increase engagement and learning achievement in two 9-year-old children who had a diagnosis of autism spectrum disorder (ASD) combined with intellectual disability, language and communication impairments, and reduced adaptive skills. The study was based on an $\mathrm{ABA}^{1}$ (i.e., baseline, intervention, and post-intervention) design. The children participated in educational sessions targeting developmental and social skills (e.g., motor imitation, expressive/receptive language, spontaneous requests). The results indicated that interacting with a social robot increased engagement and goal achievement in one of the children, and only goal achievement in the other. Those results were deemed to be promising and to support the implementation of more robust research protocols in the area.

Yu et al. (2018) assessed whether speech-language pathologists (SLPs) would be able to deliver hands-free visual supports via the Echo Show ${ }^{\mathrm{TM}}$, a voice-activated intelligent personal assistant with a touch screen displaying graphic content (e.g., photographs and videos). The study involved five participants, that is, five SLPs employed in an outpatient pediatric hospital, and investigated three questions concerning (a) the ability of the SLPs to reliably use a specific (carrier) phrase to retrieve visual supports on the Echo Show ${ }^{\mathrm{TM}}$, (b) the ability of the Echo Show ${ }^{\mathrm{TM}}$ to reliably retrieve personalized visual supports, and (c) the view of the SLPs about the usability and potential impact of the Echo Show ${ }^{\mathrm{TM}}$ within a clinical context. The results to all three questions were encouraging. The authors discussed the potential implications of those results for clinical work with individuals with ASD and stressed the need for research testing the technology directly with individuals with ASD.

Lancioni et al. (2018a) evaluated a smartphone-based program to enable five participants with mild to moderate intellectual disability, visual and/or motor impairments, and poor speech to independently access leisure and communication activities. Two Samsung Galaxy A3 smartphone devices were used for the program. The participants could make their activity requests by placing mini objects or pictures (which represented those activities and were fitted with frequency code labels) on one of the two smartphones. This smartphone read the code labels and verbalized the related activity requests. Request verbalizations activated the S-voice of the second smartphone, which opened the matching leisure activity files or telephone contacts for the participants. All participants used the program successfully and spent most of the session time busy with independently accessed leisure and communication activities.

Cannella-Malone et al. (2018) pursued two specific objectives. The first objective was to examine the effectiveness of an eye gaze preference assessment using eye gaze technology to identify highly attractive (i.e., potentially reinforcing) stimuli for three adolescents with severe intellectual and physical disabilities. The second objective was to determine the reliability of the eye gaze assessment results. Such an objective was pursued by using an $\mathrm{ABCACB}$ design, in which A represented baseline phases while $\mathrm{B}$ and $\mathrm{C}$ represented intervention phases. During the $\mathrm{B}$ and $\mathrm{C}$, the stimuli identified (via the eye gaze technology) as having high and low preference were used contingent on participants' responding, respectively. Data showed that during baseline, the participants' responding was zero or near zero. During the B phases, the participants' responding was regularly higher than that observed during the $\mathrm{C}$ phases, thus indicating that the eye gaze's discrimination between high- and low-preference stimuli was reliable.

Martinez et al. (2018) devoted the first part of their article to examining the common sequelae of traumatic brain injury (TBI), which is the leading source of disability and death among children. Indeed, they described the negative/ disabling impact that TBI has on (a) cognitive dimensions such as attention, language, and executive function, (b) emotional dimensions such as anxiety and depression, and (c) general school achievement. In the second part of the article, the authors stressed that assistive technology is a key resource for children with traumatic brain injury and can help them build compensatory strategies and increase the likelihood of living independent lives. In line with the above, the authors provided a list of various assistive technology resources for each of the main problem areas characterizing those children. For example, technology resources such as voice recorders, voice recorder software for iPod Touch or Windows Mobile, as well as cue cards and colored index cards were listed as tools to curb the disabling effects of TBI on memory.

Lancioni et al. (2018b) reported two studies with individuals in a minimally conscious state (MCS). The first study extended the assessment of a microswitch-based intervention program for helping MCS individuals increase functional responding as a way to control environmental stimulation. Ten participants were included. The second study compared the effects of the microswitch-aided intervention program with those of typical environmental stimulation strategies in terms of participants' alertness and involvement. Eight of the 10 participants involved in the first study were also included in the second study. The results of the first study confirmed the appropriateness of a microswitch-aided intervention program for supporting adaptive/functional responding and stimulation control in MCS individuals. The results of the second study 
indicated that a microswitch-aided intervention program may be more effective than basic and elaborate stimulation strategies in promoting participants' alertness and involvement.

In conclusion, the articles included in this special issue concern a variety of objectives, involve different types of participants, focus on different disabilities, and cover different technology solutions relevant for education, rehabilitation, and care contexts. Those articles can be considered helpful for the specific suggestions and answers they provide to the reader and for their contribution to the general debate on assistive technology. Such debate is critical to (a) advance our knowledge in the area, (b) explore the possibility of using specifically arranged mainstream technology devices to support individuals with different disabilities and needs, and (c) develop a new range of intervention programs that employ the technology more efficiently and pursue the achievement of greater objectives.

\section{Compliance with Ethical Standards}

Conflict of Interest The author declares that he has no conflict of interest.

Ethical Approval The research does not involve any human participants and/or animal.

Informed Consent The research does not involve any human participants and/or animal.

\section{References}

Beale, I. L., Ramloll, R., Rose, G. J., \& Malone, J. W. (2018). A virtual world for learning skills of independent living: a formative evaluation. Advances in Neurodevelopmental Disorders, 2.

Boyd, H. C., Evans, N. M., Orpwood, R. D., \& Harris, N. D. (2017). Using simple technology to prompt multistep tasks in the home for people with dementia: an exploratory study comparing prompting formats. Dementia (London), 16, 424-442.

Cannella-Malone, H. I., Schmidt, E. K., \& Bumpus, E. C. (2018). Assessing preference using eye gaze technology for individuals with significant intellectual and physical disabilities. Advances in Neurodevelopmental Disorders, 2.

Damianidou, D., Foggett, J., Arthur-Kelly, M., Lyons, G., \& Wehmeyer, M. L. (2018). Effectiveness of technology types in employment-related outcomes for people with intellectual and developmental disabilities: an extension meta-analysis. Advances in Neurodevelopmental Disorders, 2.

Davies, D. K., Stock, S. E., Herold, R. G., \& Wehmeyer, M. L. (2018). GeoTalk: a GPS-enabled portable speech output device for people with intellectual disability. Advances in Neurodevelopmental Disorders, 2.

Desideri, L., Negrini, M., Malavasi, M., Tanzini, D., Rouame, A., Cutrone, M. C., Bonifacci, P., \& Hoogerwerf, E.-J. (2018). Using a humanoid robot as a complement to interventions for children with autism spectrum disorder: a pilot study. Advances in Neurodevelopmental Disorders, 2.

Kerkhof, Y. J., Graff, M. J., Bergsma, A., de Vocht, H. H., \& Dröes, R. M. (2016). Better self-management and meaningful activities thanks to tablets? Development of a person-centered program to support people with mild dementia and their carers through use of hand-held touch screen devices. International Psychogeriatrics, 28, 1917-1929.

Lancioni, G. E., \& Singh, N. N. (Eds.). (2014). Assistive technologies for people with diverse abilities. New York: Springer.

Lancioni, G. E., Bosco, A., Olivetti Belardinelli, M., Singh, N. N., O'Reilly, M. F., Sigafoos, J., et al. (2014). Technology-based intervention programs to promote stimulation control and communication in post-coma persons with different levels of disability. Frontiers in Human Neuroscience, 8, 48. https://doi.org/10.3389/ fnhum.2014.00048.

Lancioni, G., Singh, N., O’Reilly, M., Sigafoos, J., D’Amico, F., Pinto, K., et al. (2017). A technology-aided program for helping persons with Alzheimer's disease perform daily activities. Journal of Enabling Technologies, 11, 85-91.

Lancioni, G. E., O'Reilly, M. F., Sigafoos, J., Campodonico, F., Perilli, V., Alberti, G., Ricci, C., \& Miglino, O. (2018a). A modified smartphone-based program to support leisure and communication activities in people with multiple disabilities. Advances in Neurodevelopmental Disabilities, 2. https://doi.org/10.1007/ s41252-017-0047-z.

Lancioni, G. E., O’Reilly, M. F., Sigafoos, J., D’Amico, F., Buonocunto, F., Devalle, G., Trimarchi, P. D., Navarro, J., \& Lanzilotti, C. (2018b). A further evaluation of microswitch-aided intervention for fostering responding and stimulation control in persons in a minimally conscious state. Advances in Neurodevelopmental Disorders, 2. https://doi.org/10.1007/s41252-018-0064-6.

Lancioni, G., Singh, N., O’Reilly, M., Sigafoos, J., Alberti, G., Chiariello, V., et al. (2018c). A smartphone-based technology package to support independent activity in people with intellectual disability and blindness. Internet Technology Letters, 2018, e34. https://doi.org/10. 1002/it12.34.

Lancioni, G. E., Singh, N. N., O’Reilly, M. F., Sigafoos, J., D’Amico, F., Pinto, K., et al. (2018d). Promoting supported ambulation in persons with advanced Alzheimer's disease: a pilot study. Disability and Rehabilitation: Assistive Technology, 13, 101-106.

Martinez, A. P., Scherer, M., \& Tozser, T. (2018). Traumatic brain injury (TBI) in school based populations: common sequelae and assistive technology interventions. Advances in Neurodevelopmental Disabilities, 2.

Mechling, L. C. (2006). Comparison of the effects of three approaches on the frequency of stimulus activations, via a single switch, by students with profound intellectual disabilities. Journal of Special Education, 40, 94-102.

Myers, R. E., Karazsia, B. T., Kim, E., Jackman, M. M., McPherson, C. L., \& Singh, N. N. (2018). A telehealth parent-mediated mindfulness-based health wellness intervention for adolescents and young adults with intellectual and developmental disabilities. Advances in Neurodevelopmental Disabilities, 2.

Puanhvuan, D., Khemmachotikun, S., Wechakarn, P., Wijarn, B., \& Wongsawat, Y. (2017). Navigation-synchronized multimodal control wheelchair from brain to alternative assistive technologies for persons with severe disabilities. Cognitive Neurodynamics, 11, 117-134.

Schlosser, R. W., O’Brien, A., Yu, C., Abramson, J., Allen, A. A., Flynn, S., \& Shane, H. C. (2017). Repurposing everyday technologies to provide just-in-time visual supports to children with intellectual disability and autism: a pilot feasibility study with the Apple Watch ${ }^{\circledR}$. International Journal of Developmental Disabilities, 63, 221-227. 
Wang, R. H., Sudhama, A., Begum, M., Huq, R., \& Mihailidis, A. (2017). Robots to assist daily activities: views of older adults with Alzheimer's disease and their caregivers. International Psychogeriatrics, 29, 67-79.

Williamson, B., Aplin, T., de Jonge, D., \& Goyne, M. (2017). Tracking down a solution: exploring the acceptability and value of wearable GPS devices for older persons, individuals with a disability, and their support persons. Disability and Rehabilitation Assistive Technology, 12, 822-831.
Wu, P. F., Wheaton, J. E., \& Cannella-Malone, H. I. (2016). Effects of video prompting and activity schedules on the acquisition of independent living skills of students who are deaf and have developmental disabilities. Education and Training in Autism and Developmental Disabilities, 51, 366-378.

Yu, C., Shane, H., Schlosser, R. W., O’Brien, A. O., Allen, A., Abramson, J., \& Flynn, S. (2018). An exploratory study of speech-language pathologists using the Echo Show ${ }^{\mathrm{TM}}$ to deliver visual supports. Advances in Neurodevelopmental Disorders, 2. 